Tecno Lógicas

ISSN 0123-7799

Vol. 17, No. 33, pp. 43-54

Julio-diciembre de 2014
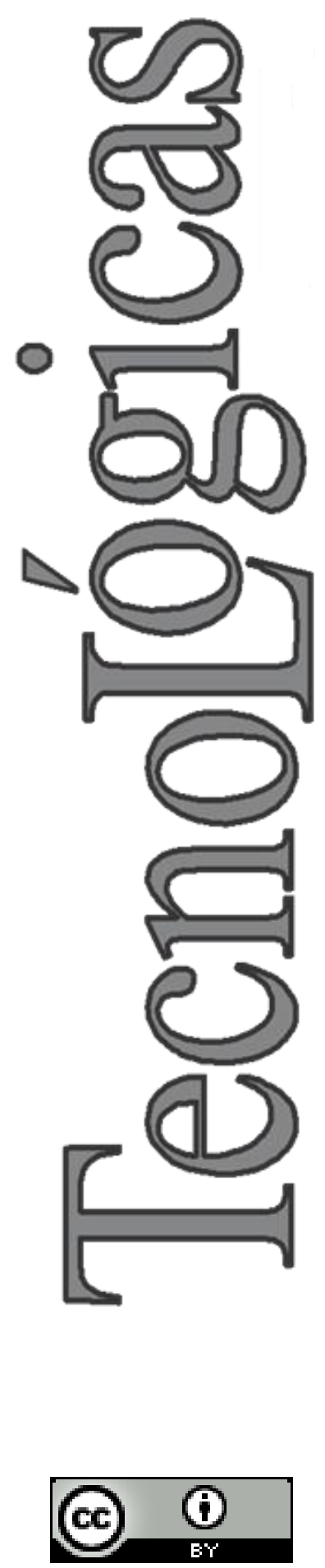

\section{Uso de la cascarilla de arroz para la remoción de azul de metileno en columnas de lecho empacado}

\author{
Use of rice husk for the removal of \\ methylene blue in fixed-bed columns
}

\author{
Yurany A. Villada-Villada ${ }^{1}$, \\ Angelina Hormaza-Anaguano² y Natalia Casis ${ }^{3}$
}

Recibido: 3 de marzo de 2014, Aceptado: 2 de mayo de 2014

Cómo citar / How to cite

Y. A. Villada-Villada, A. Hormaza-Anaguano y N. Casis, "Uso de la cascarilla de arroz para la remoción de azul de metileno en columnas de lecho empacado", Tecno Lógicas, vol. 17, no. 33, pp. 43-54, 2014.

1 Ingeniera Química, Facultad de Minas, Universidad Nacional de Colombia, Medellín-Colombia, yavillad@unal.edu.co

2 Ph.D. en Ciencias Naturales, Profesor Asociado, Universidad Nacional de Colombia, Medellín-Colombia, ahormaza@unal.edu.co

3 Ph.D. en Tecnología Química, Instituto de Desarrollo Tecnológico para la Industria Química - INTEC, Universidad Nacional del Litoral, Santa Fe-Argentina, ncasis@santafe-conicet.gov.ar 


\section{Resumen}

Este trabajo muestra la utilización de la cascarilla de arroz para la remoción del colorante catiónico azul de metileno bajo sistema continuo. Un diseño factorial $2^{3}$, con puntos centrales y distribución aleatoria, fue implementado para evaluar la correlación de los factores experimentales en el proceso de adsorción. Las variables consideradas fueron $\mathrm{pH}$, tamaño de partícula, presencia salina, velocidad de entrada, concentración inicial del colorante y altura de columna. Las muestras fueron analizadas a intervalos definidos de tiempo. La cantidad del colorante removido fue cuantificado por medio de espectroscopia Ultravioleta - Visible. Los modelos de Adams-Bohart, Thomas y BDST (Bed-depht/service time analysis) fueron utilizados para predecir las curvas de ruptura usando regresión no lineal y establecer los parámetros característicos del proceso. Se encontró que la transferencia de colorante al material adsorbente se favorece a $\mathrm{pH}$ básico, menor tamaño de partícula, flujo y concentración baja y mayor altura. El diseño de experimentos estableció que la concentración inicial de colorante y la altura del lecho fueron los factores más significativos. Con respecto a los modelos, el de Thomas presentó el mejor ajuste para la descripción de las curvas de ruptura a las condiciones experimentales y el de Adams-Bohart resultó adecuado para el comportamiento dinámico limitado a la parte inicial. Finalmente, el modelo BDST mostró una buena correlación y permitió establecer que la altura es determinante para el escalado del proceso.

\section{Palabras clave}

Adsorción en columna, altura de lecho, colorante catiónico, curva de ruptura, diseño de experimentos.

\section{Abstract}

This work shows the use of rice husk in the removal of cationic dye methylene blue on continuous system. A factorial design $2^{3}$ with center points and random distribution was implemented to evaluate the correlation of the experimental factors in the adsorption process. The considered variables were $\mathrm{pH}$, particle size, salt presence, flow rate, dye initial concentration, and bed depth. The samples were analyzed in defined time intervals. The amount of removed dye was quantified by UV spectroscopy - Visible. Adams-Bohart, Thomas and BDST (Bed-depht/service time analysis) models were used to predict the breakthrough curves using non-linear regression and establish the characteristic parameters of the process. It was found that the transference of dye toward the adsorbent is favored by a basic $\mathrm{pH}$, a small particle size, low flow rate and dye concentration, and high bed depth. The design of experiments established that the initial dye concentration and the bed depth were the most significant factors. Regarding the models, the Thomas provided the best fit to describe the breakthrough curves in experimental conditions and Adams-Bohart was found suitable for dynamic behavior limited to the initial part. Finally, BDST model exhibited a good correlation and allowed to establish that bed depth is a determinant factor for scaling process.

\section{Keywords}

Adsorption in fixed-bed, bed depth, cationic dye, breakthrough curve, experiments design. 


\section{INTRODUCCIÓN}

La contaminación de las fuentes de agua debida a la descarga de efluentes industriales coloreados crece aceleradamente como consecuencia de la mínima regulación ambiental y del aumento de empresas que utilizan colorantes en sus diferentes procesos. Sus principales manifestaciones se evidencian tanto a nivel estético, por el deterioro visual del entorno, como por el desequilibrio dinámico del ecosistema, donde en particular, la disminución de oxígeno, afecta el normal desarrollo de múltiples procesos para la preservación de la biota acuática [1].

Dentro de los métodos físicos más ampliamente utilizados para el tratamiento de colorantes en solución, la adsorción ha mostrado ser un proceso eficiente, de fácil implementación y atractivo por sus ventajas adicionales de retención del contaminante sin fraccionamiento y posibilidad de reúso del material adsorbente hasta alcanzar su grado de saturación. Si bien es cierto que el carbón activado ofrece una elevada eficiencia en la retención de contaminantes, su costosa adquisición [2] ha llevado a la búsqueda de adsorbentes alternativos, de amplia disponibilidad y mínimo precio, tales como los residuos agroindustriales [3], [4].

$\mathrm{Al}$ respecto, múltiples investigaciones han reportado el uso de diferentes tipos de residuos agrícolas tales como cascarilla de arroz [5], tuza de maíz [6], [7], cáscaras de banano [8] y residuos de flores [9], entre otros para la remoción de colorantes de naturaleza catiónica y aniónica. Las propiedades adsorbentes de estos materiales son atribuidas en particular a su elevado contenido de lignina, celulosa y hemicelulosa, cuyos grupos funcionales posibilitan la interacción con los contaminantes disueltos [10].

El azul de metileno, AM, es un colorante de naturaleza catiónica, usado comúnmente en la industria textil para la tinción de algodón, lana, madera, papel y seda.
Sus características de toxicidad hacen que sea considerado como un compuesto con efectos secundarios perjudiciales para la salud, ya que incrementa el ritmo cardíaco, produce alteraciones gástricas y nerviosas, así como también puede inducir la aparición de enfermedades cutáneas como la cianosis e ictericia [5].

Por otro lado, el residuo agrícola cascarilla de arroz, CA, ha sido evaluado como adsorbente alternativo en la remoción tanto de metales, entre ellos Cr(VI), As(V), $\mathrm{Au}, \mathrm{Cu}(\mathrm{II}), \mathrm{Pb}(\mathrm{II}), \mathrm{Fe}(\mathrm{II}), \mathrm{Mn}(\mathrm{II}), \mathrm{Zn}(\mathrm{II})$ y Cd(II), [11], [12], como de colorantes en solución [13]. Su reutilización en los procesos de adsorción ha sido estimada en un promedio de cuatro ciclos. En la mayoría de casos, ha sido empleado con tratamiento térmico o en forma de carbones activados, mostrando una retención relativamente baja con los colorantes azul sandolan, naranja sandocril, azul foron y amarillo ácido 36. Cabe resaltar sin embargo, la gran afinidad reportada para la safranina y el azul de metileno [14].

Cabe señalar que la información derivada de un proceso de adsorción bajo sistema discontinuo ofrece información valiosa sobre la eficiencia del sistema coloranteadsorbente, y usualmente representa una etapa exploratoria en la definición de un adsorbente adecuado para un determinado colorante. No obstante, dicha información es insuficiente al momento de magnificar el proceso de tratamiento. Por ello y como preámbulo para un escalado del proceso de adsorción se requiere llevar a cabo el estudio bajo sistema continuo de la pareja colorante-adsorbente de interés.

Para el caso del colorante AM, se dispone sólo de una investigación previa sobre su remoción mediante columnas empacadas con el residuo agrícola CA [15]. Han et al., se limitaron a la utilización de dos modelos matemáticos, Thomas y BDST, para el ajuste de los datos experimentales. Por otro lado, usaron flujos de entrada de 3,4 - 8,2 $\mathrm{mL} \min ^{-1} \mathrm{y}$ un diámetro interno de columna de $1,5 \mathrm{~cm}$, es decir, condiciones 
restringidas para un posterior proceso de escalado. Adicionalmente, no consideraron la implementación de un diseño estadístico, con la subsecuente pérdida de información de la influencia y correlación de las variables de estudio para la optimización del proceso.

En este marco de referencia, el propósito del presente trabajo es verificar la posibilidad de utilizar CA para la adsorción de AM bajo sistema continuo, y determinar la interacción de las variables mediante un diseño estadístico de experimentos, que señale la importancia de cada factor hacia la optimización y escalado del proceso. Por ello, el objetivo de esta investigación fue llevar a cabo la evaluación de las principales variables, $\mathrm{pH}$ de la solución, tamaño de partícula, velocidad de entrada, concentración inicial del colorante, y altura del lecho en la remoción en continuo del colorante AM sobre CA. Los modelos de AdamsBohart, Thomas y BDST, fueron utilizados como base para la estimación de los parámetros característicos del proceso de adsorción [16].

\subsection{Modelos matemáticos implementados}

El rendimiento de una columna de lecho fijo se representa a través del concepto de la curva de ruptura. El tiempo de ruptura y la forma de la curva son características importantes tanto para la determinación de operación como para el establecimiento de la respuesta dinámica de la columna [17]. Así, el comportamiento del colorante, impregnado en el lecho de CA, se expresa en términos de la concentración inicial y del tiempo, $\mathrm{Ct} / \mathrm{Co}$, como una función del tiempo o el volumen del efluente a una altura dada, generando de esta manera la curva respectiva.

\subsection{Modelo de Adams-Bohart}

Este modelo es usado para describir la parte inicial de las curvas de ruptura y se expresa a través de (1).
$\frac{C_{t}}{C_{0}}=\exp \left(k_{A B} C_{0} t-k_{A B} N_{0} \frac{Z}{F}\right)$

Donde $k_{A B}$ es la constante cinética (L $\left.\mathrm{mg}^{-1} \mathrm{~min}^{-1}\right), F$ es la velocidad lineal calculada dividiendo el flujo por el área transversal de la columna (cm $\left.\mathrm{min}^{-1}\right), Z$ es la altura del lecho en la columna y $N_{0}$ es la concentración de saturación $\left(\mathrm{mg} \mathrm{L}^{-1}\right)$. Los parámetros característicos se determinan a partir de la gráfica de Ct/Co vs $t$, a una altura de lecho y flujo de entrada dado, mediante una regresión no-lineal.

\subsection{Modelo de Thomas}

Es uno de los métodos ampliamente usado en la representación y análisis de los datos obtenidos a partir de ensayos en columnas. Se utiliza para conocer la máxima capacidad de adsorción del material adsorbente y la constante cinética [16]. Este modelo se describe mediante (2).

$\frac{C_{t}}{C_{0}}=\frac{1}{1+\exp \left(k_{T h} q_{0} x / v-k_{T h} C_{0} t\right)}$

Donde $k_{T h}$ es la constante de Thomas (mL $\left.\min ^{-1} \mathrm{mg}^{-1}\right) ; q_{0}$ es la cantidad de AM por gramo de adsorbente ( $\left.\mathrm{mg} \mathrm{g}^{-1}\right) ; x$ es la cantidad de adsorbente en la columna (g); $C_{0}$ es la concentración inicial del efluente de AM (mg L $\left.{ }^{-1}\right) ; C_{t}$ es la concentración del efluente en el tiempo $t\left(\mathrm{mg} \mathrm{L}^{-1}\right)$ y $v$ es el flujo de entrada $\left(\mathrm{mL} \mathrm{min} \mathrm{m}^{-1}\right)$. El coeficiente cinético $k_{T h}$ y la capacidad de adsorción de la columna, $q_{0}$, pueden ser determinados a partir de la gráfica de $C t / C o$ vs $t$, a una velocidad de entrada dada, mediante una regresión no-lineal.

\subsection{Modelo Bed-depth/ service time (BDST)}

Este modelo permite predecir la relación entre la altura de la columna, $Z$, y el tiempo de residencia, en términos de las concentraciones y parámetros de adsorción del proceso. Está determinado por (3). 
$t=\frac{N_{O}}{C_{O}} Z-\frac{1}{K_{a} C_{a}} \ln \left(\frac{C_{O}}{C_{T}}-1\right)$

Donde $C_{t}$ es la concentración de la solución en el tiempo (mg L-1), $C_{0}$ es la concentración inicial de la solución $\left(\mathrm{mg} \mathrm{L}^{-1}\right), F$ es el flujo de entrada $\left(\mathrm{cm} \mathrm{min}{ }^{-1}\right), N_{o}$ es la capacidad de adsorción ( $\left.\mathrm{mg} \mathrm{g}^{-1}\right), K_{a}$ es la

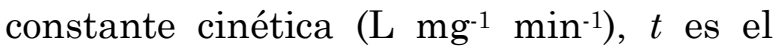
tiempo (min) y $Z$ es la altura de lecho en la columna (cm), [16]. En una gráfica de t vs $\mathrm{Z}$, se puede determinar las variables $K_{a}, \mathrm{y}$ $N_{o}$ mediante un análisis de regresión lineal.

\subsection{Análisis de error}

Se realiza un análisis con el coeficiente de correlación $\left(\mathrm{R}^{2}\right)$ y el error estadístico (SS), expresado a través de (4), para calcular la probabilidad de error y estimar la validez de cada modelo presentado.

$S S=\sqrt{\sum \frac{\left(y_{e}-y_{c}\right)^{2}}{N}}$

Donde $y_{e}$ y $y_{c}$ corresponden al valor experimental y al calculado según el modelo respectivamente y $N$ es el número de experimentos [16].

\section{METOdOLOGíA}

\subsection{Preparación del adsorbente}

La CA fue obtenida de las agroindustrias arroceras del departamento de Córdoba y sometida a un pretratamiento que incluyó molienda, tamizado, lavado y secado. Para el estudio se seleccionaron tres tamaños de partícula entre 0,3 y 1,0 $\mathrm{mm}$. El lavado se realizó cinco veces con agua destilada y peróxido de hidrógeno (5\%). Luego, la CA fue secada en un horno de convección forzada a una temperatura de $80^{\circ} \mathrm{C}$ durante $48 \mathrm{~h}$ y conservada en recipientes herméticos de vidrio para los ensayos posteriores.

\subsection{Preparación de la solución}

Las soluciones de AM se prepararon en agua destilada a dos concentraciones, $50 \mathrm{y}$ $100 \mathrm{mg} \mathrm{L}^{-1}$ respectivamente, ajustando el valor de $\mathrm{pH}$ con alícuotas de soluciones de $\mathrm{HCl}$ e $\mathrm{NaOH}$ 0,1 M. Adicionalmente, para la evaluación de la influencia de $\mathrm{NaCl}$ en el proceso de adsorción, se prepararon las soluciones a las mismas condiciones, pero adicionando 100 g de $\mathrm{NaCl}$ por cada g de colorante utilizado; dicha relación $\mathrm{NaCl} /$ colorante se aproxima a la empleada en la industria textil para el proceso de tinción.

\subsection{Estudio de adsorción en continuo}

Los experimentos de adsorción a flujo continuo fueron realizados en una columna de vidrio, cuyas dimensiones generales fueron $2,4 \mathrm{~cm}$ de diámetro interno y $50 \mathrm{~cm}$ de altura. Una serie de experimentos fueron llevados a cabo para analizar la influencia de cada variable en el proceso, tomando como referencia las curvas de ruptura resultantes de cada ensayo.

Los parámetros evaluados fueron, influencia de $\mathrm{NaCl}$ en la solución, $\mathrm{pH}$ del medio $(2,0,6,0$ y 9,0$)$, tamaño de partícula (0,3 $\mathrm{mm}<x<1,0 \mathrm{~mm})$, concentración inicial de colorante $\left(C_{0}=50\right.$ y $\left.100 \mathrm{mg} \mathrm{L}^{-1}\right)$, flujo de entrada $\left(V=10\right.$ y $\left.20 \mathrm{~mL} \mathrm{~min}^{-1}\right)$ y altura del lecho $(Z=10,15$ y $20 \mathrm{~cm})$. La solución de AM, a una concentración determinada, fue bombeada en flujo ascendente con una bomba peristáltica marca VWR de especificación flujo medio-alto. Las muestras fueron colectadas a la salida de la columna en intervalos regulares de tiempo hasta alcanzar la saturación total del lecho.

La concentración de AM fue cuantificada por medio de espectroscopia de UV-Vis, a una longitud de onda máxima de $\lambda_{\text {máx }}=$ $665 \mathrm{~nm}$, utilizando un espectrofotómetro de 
doble haz UV-Vis Lambda 35, marca Perkin Elmer. Para determinar la influencia de la solución salina, se compararon los resultados obtenidos en las curvas de ruptura en ausencia y presencia de $\mathrm{NaCl}$, utilizando una altura de lecho de $10 \mathrm{~cm}$, una concentración inicial de $100 \mathrm{mg} \mathrm{L}^{-1} \mathrm{y}$ un flujo de entrada constante de $20 \mathrm{~mL}$ $\min ^{-1}$.

\subsection{Estimación de las curvas de ruptura}

El comportamiento del sistema AM-CA es descrito a partir del ajuste de los resultados experimentales a los modelos de Adams-Bohart, Thomas y BDST, considerando las variables, flujo de entrada a la columna, concentración inicial de colorante y altura del lecho, mediante un análisis de regresión no lineal. Además, el error estadístico y el factor de correlación fueron seleccionados como verificadores de los modelos implementados.

\subsection{Diseño estadístico de experimentos}

En particular, se utilizó como herramienta estadística un diseño factorial de cribado $2^{3}$ con puntos centrales y distribución aleatoria, con los factores, concentración inicial de colorante, velocidad de entrada a la columna y altura de lecho, cuyos niveles se detallen en la Tabla 1 . La variable de respuesta seleccionada fue el porcentaje de remoción a un tiempo de saturación dado. El análisis fue llevado a cabo haciendo uso de la versión libre del software Statgraphics Centurion XV.II 15.2.06.

Tabla 1. Valores para el diseño de cribado $2^{3}$ del sistema AM-CA. Fuente: Autores

\begin{tabular}{lccc}
\hline Niveles & $\begin{array}{c}\text { Concentración } \\
{\left[\mathrm{mg} \mathrm{L}^{-1}\right]}\end{array}$ & $\begin{array}{c}\text { Velocidad de } \\
\text { entrada } \\
{\left[\mathrm{mL} \mathrm{min}^{-1}\right]}\end{array}$ & $\begin{array}{c}\text { Altura del } \\
\text { lecho }[\mathrm{cm}]\end{array}$ \\
\hline Bajo & 50 & 10 & 10 \\
Central & 75 & 15 & 15 \\
Alto & 100 & 20 & 20 \\
\hline
\end{tabular}

\section{RESULTADOS Y DISCUSIÓN}

\subsection{Análisis del diseño de experimentos de cribado}

El diseño factorial $2^{3}$ se utilizó para determinar las variables más influyentes y $\mathrm{su}$ interacción en el proceso de adsorción en continuo del sistema AM-CA y de esta forma, establecer los intervalos más adecuados de cada una de las variables de estudio. Los resultados del diseño de cribado son presentados en la Fig. 1.

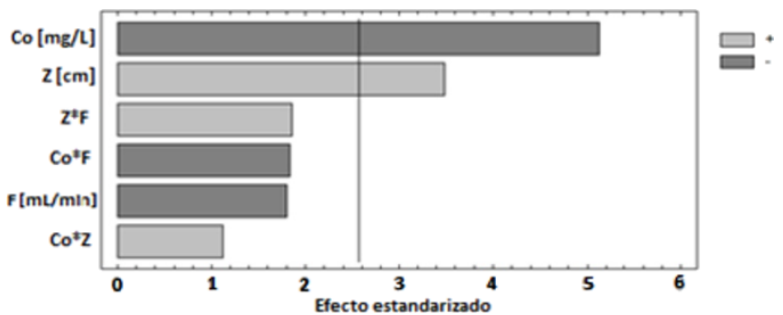

a)

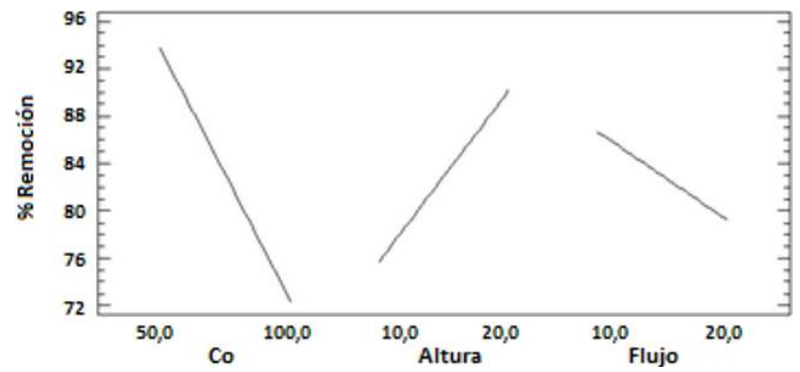

b)

Fig. 1. a) Diagrama de Pareto estandarizado para el porcentaje de remoción del diseño de cribado $2^{3}$ del sistema AM-CA, b) gráfico de efectos principales para el diseño factorial. Fuente: Autores

Considerando un nivel de confianza del 95,0\%, en el diagrama de Pareto, Fig. 1a, se observa que el factor más significativo es la concentración del colorante con un efecto negativo, es decir, que a medida que la concentración aumenta, el porcentaje de remoción disminuye. En segundo lugar de significancia se encuentra la altura de la columna, con efecto positivo, esto es, un aumento de este factor conlleva a un incremento proporcional en el porcentaje de remoción del colorante de interés. 
Por otro lado, el factor velocidad de entrada y su interacción, tanto con la concentración de colorante como con la altura de columna, no presenta una significancia en el porcentaje de remoción del AM sobre CA. En la Fig. 1b, se corrobora lo anteriormente descrito, donde el porcentaje de adsorción a un tiempo de saturación determinado se ve favorecido a alturas mayores, menores concentraciones de colorante y bajas velocidades en el flujo de entrada.

Además, cabe señalar que el modelo permitió alcanzar una remoción máxima del $95,71 \%$ a una concentración de colorante de $50 \mathrm{mg} \mathrm{L}^{-1}$, una altura de lecho de 20 $\mathrm{cm}$ y a una velocidad de entrada de $10 \mathrm{~mL}$ min $^{-1}$, evaluado a un tiempo de saturación de $3 \mathrm{~h}$, expresada en (5).

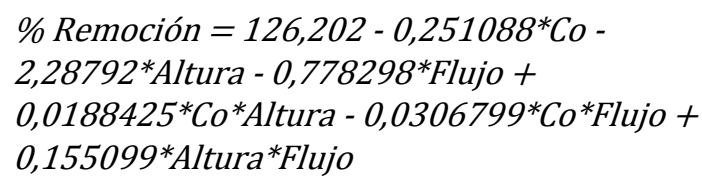

\subsection{Efecto del pH de la solución inicial en la curva de ruptura}

La influencia de esta variable en la adsorción del AM sobre CA se evaluó a los siguientes valores de $\mathrm{pH}=2,0,6,0$ y 9,0 . En la Fig. 2, se observa que un incremento de $\mathrm{pH}$ conduce a un desplazamiento de izquierda a derecha en las curvas de ruptura, indicando que el tiempo requerido para alcanzar la saturación del lecho es mayor y por tanto, aumenta la cantidad removida de AM.

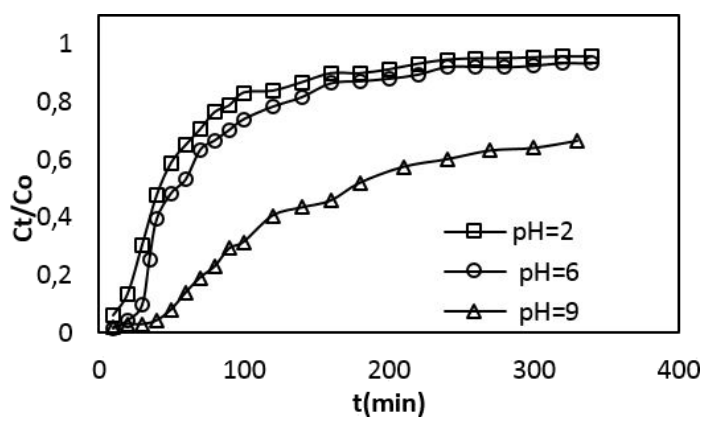

Fig. 2. Curvas de ruptura. Análisis del efecto de $\mathrm{pH}$ en la adsorción de AM-CA $\left(\mathrm{C}_{0}=100 \mathrm{mg} \mathrm{L}^{-1}, \mathrm{~V}=20 \mathrm{~mL} \mathrm{~min}^{-1}\right.$, $\mathrm{Z}=10 \mathrm{~cm})$. Fuente: Autores
Es decir, que la remoción del colorante de estudio se ve favorecida en un medio básico, en este caso particular, a un $\mathrm{pH}=$ 9,0 , valor seleccionado para los ensayos con las variables restantes. Así por ejemplo, al comparar el proceso a un tiempo de 240 min, se aprecia que a un $\mathrm{pH}=9,0$ el lecho solo ha alcanzado una saturación del 59\%, en tanto que a valores de $\mathrm{pH}=6,0$ y 2,0 , la saturación es del orden de 92 y $94 \%$ respectivamente.

Diferentes razones pueden argumentarse para explicar el aumento de la adsorción del AM sobre CA a medida que incrementa el valor del $\mathrm{pH}$ del medio. Principalmente, las partículas de la superficie del adsorbente pueden estar cargadas negativamente, generando así un gran número de sitios activos de carga negativa, que por afinidad electrónica favorecen las atracciones electrostáticas con las cargas positivas del colorante catiónico AM. Por el contrario, valores de $\mathrm{pH}$ ácidos pueden conducir a la formación de sitios activos cargados positivamente sobre la CA, dando lugar a un aumento de las fuerzas de repulsión entre las cargas del colorante y la superficie del adsorbente.

\subsection{Influencia del tamaño partícula en la curva de ruptura}

La influencia del tamaño de partícula del adsorbente CA se evaluó utilizando tres tamaños, $0,3 \mathrm{~mm}<x<0,5 \mathrm{~mm}, 0,5 \mathrm{~mm}<x$ $<0,7 \mathrm{~mm}$ y $0,7 \mathrm{~mm}<x<1,0 \mathrm{~mm}$ respectivamente. Como se puede apreciar en la Fig. 3, a un tiempo de $270 \mathrm{~min}$ y con un tamaño de partícula de $0,3 \mathrm{~mm}<x<0,5$ $\mathrm{mm}$, la columna presenta una saturación de $\mathrm{AM}$ del $63 \%$, en tanto que a tamaños de partícula de $0,5 \mathrm{~mm}<x<0,7 \mathrm{~mm}$ y 0,7 $\mathrm{mm}<x<1,0 \mathrm{~mm}$, el porcentaje de saturación del colorante es del orden de 83 y 94\% respectivamente, señalando que un tamaño de partícula mayor da lugar a una menor cantidad de colorante removido debido a la considerable saturación alcanzada en com- 
paración con la registrada para el menor tamaño de partícula.

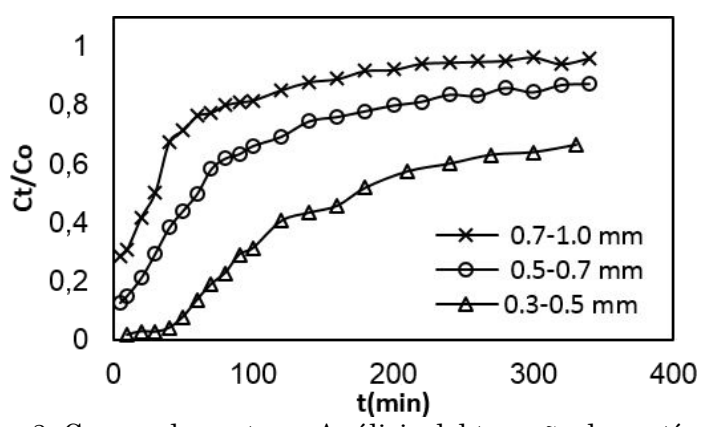

Fig. 3. Curvas de ruptura. Análisis del tamaño de partícula en la adsorción de AM-CA $\left(\mathrm{C}_{0}=100 \mathrm{mg} \mathrm{L}{ }^{-1}, \mathrm{~V}=20 \mathrm{~mL} \mathrm{~min}^{1}\right.$,

$\mathrm{Z}=10 \mathrm{~cm}, \mathrm{pH}=9,0)$. Fuente: Autores

Así, la remoción del sistema AM-CA se ve favorecida a un tamaño de partícula menor, dado que existe una mayor área superficial expuesta del adsorbente, lo cual incrementa el área de contacto entre el adsorbente y el adsorbato. Por esta razón, el tamaño de partícula $0,3 \mathrm{~mm}<x<0,5$ $\mathrm{mm}$ fue seleccionado para la evaluación de las variables restantes.

\subsection{Influencia de la solución de NaCI}

El efecto de la solución salina en la remoción de AM sobre CA es mostrado en la Fig. 4., donde se presentan las curvas de ruptura en ausencia y presencia de $\mathrm{NaCl}$.

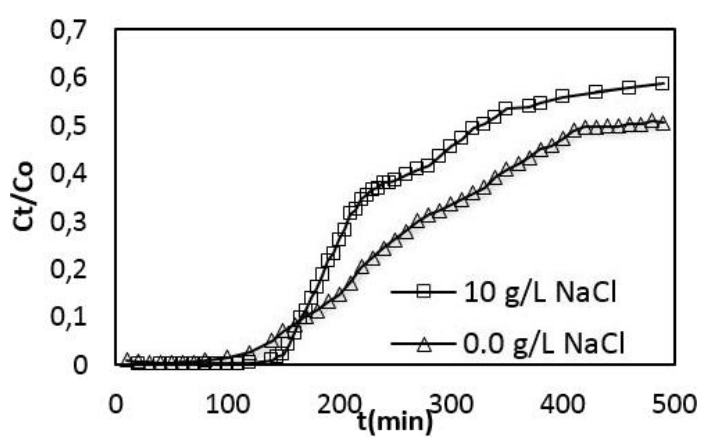

Fig. 4. Curvas de ruptura. Análisis de la influencia de $\mathrm{NaCl}$ en la adsorción de AM-CA $\left(\mathrm{C}_{0}=100 \mathrm{mg} \mathrm{L}^{-1}, \mathrm{~V}=20 \mathrm{~mL} \mathrm{~min}^{1}\right.$, $\mathrm{Z}=10 \mathrm{~cm}, \mathrm{pH}=9,0$, tamaño de partícula entre 0,3-0,5 $\mathrm{mm})$. Fuente: Autores

De forma general, un contenido de sal, en este caso $10 \mathrm{~g} \mathrm{~L}^{-1}$, conduce a un desplazamiento en la curva de ruptura de derecha a izquierda, señalando que una menor cantidad de colorante es retenida debido a que la saturación es alcanzada en un tiempo menor, en contraste con el requerido para el proceso en ausencia de sal.

Si se considera un tiempo de $280 \mathrm{~min}$, se observa que la presencia de $\mathrm{NaCl}$ genera una saturación del lecho del $41 \%$, mientras que en su ausencia se registra una saturación del 31\%. Lo anterior, permite confirmar que la retención del colorante AM se ve favorecida en ausencia de $\mathrm{NaCl}$. Sin embargo, en busca de simular condiciones más cercanas a los efluentes coloreados reales, los ensayos fueron realizados considerado la presencia de $\mathrm{NaCl}$ en la solución.

La menor remoción del AM en presencia de $\mathrm{NaCl}$ se atribuye al efecto competitivo entre los iones del colorante y los cationes metálicos de la sal por los sitios activos cargados negativamente sobre la superficie del adsorbente. De esta forma, los cationes $\mathrm{Na}^{+}$ocupan dichos sitios, reduciendo la posibilidad de interacción entre el AM y el adsorbente.

\subsection{Efecto de la altura de la columna}

La influencia de esta variable $(10,15$ y $20 \mathrm{~cm}$ ) sobre el sistema AM-CA, se presenta en la Fig. 5a, donde se aprecia que un incremento en el tamaño del lecho, conduce a un mayor tiempo de contacto entre el AM y la CA, de tal forma, que una mayor cantidad del colorante es retenido por el adsorbente. Lo anterior, permite un mejor aprovechamiento en la zona de transferencia de masa, retardando el tiempo de saturación de la columna y posibilitando de esta manera, el tratamiento de grandes volúmenes de efluentes contaminados.

\subsection{Efecto de la velocidad de entrada y de la concentración inicial de colorante}

En la Fig. 5b. se observa la rápida saturación de la curva de ruptura al utilizar un flujo alto $\left(20 \mathrm{~mL} \mathrm{~min}^{-1}\right)$. Por el contrario, un flujo bajo $\left(10 \mathrm{~mL} \mathrm{~min}^{-1}\right)$ da lugar a una saturación lenta y con ello a una mayor 
retención de $\mathrm{AM}$ en el lecho empacado. Estos resultados se atribuyen a la transferencia de masa del sistema, pues flujos altos incrementan tanto la velocidad de transferencia como la cantidad de colorante adsorbido por gramo de adsorbente, generando un menor tiempo de contacto entre colorante-adsorbente y por tanto, una rápida saturación del lecho.
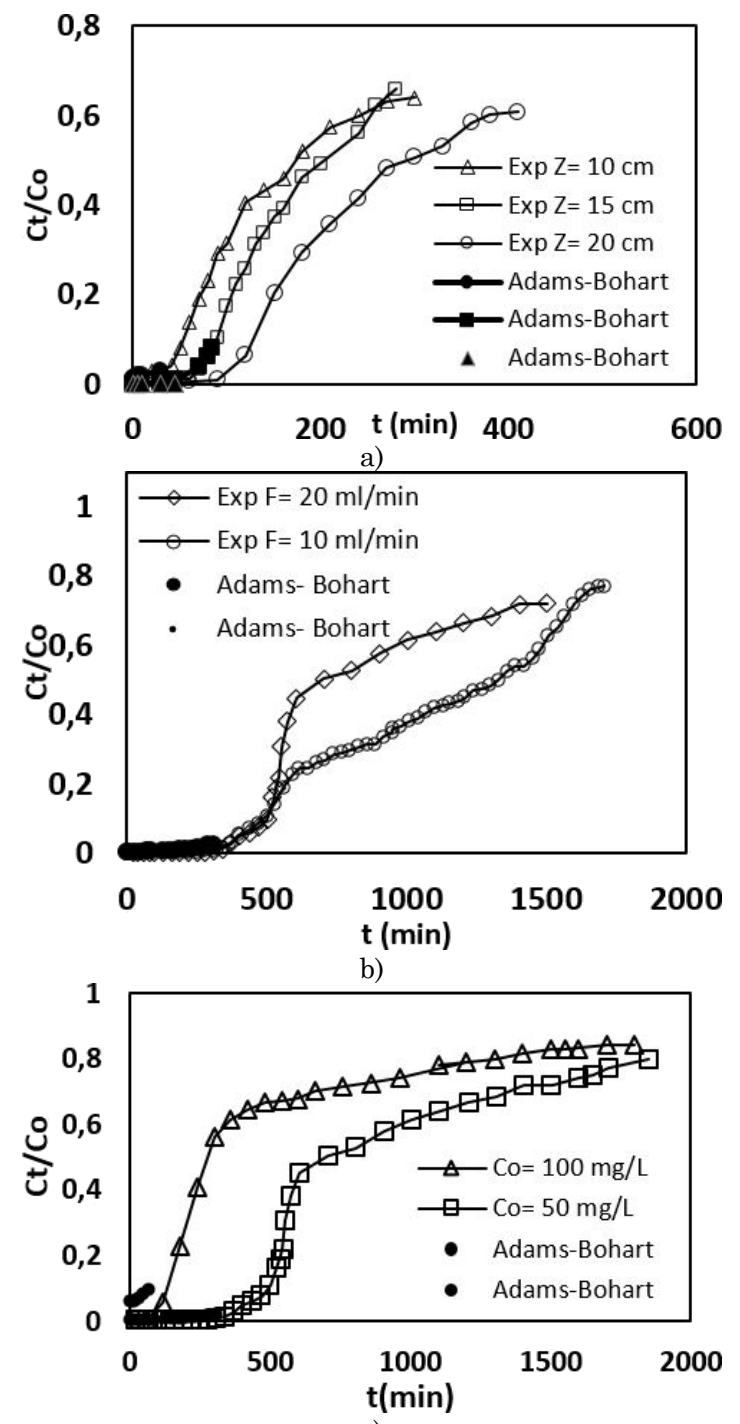

Fig. 5. Curvas de ruptura. Modelo de Adams-Bohart aplicado al sistema AM-CA. a) Efecto de la altura, b) efecto de la concentración inicial y c) efecto de la velocidad de entrada ( $\mathrm{pH}=9,0$, tamaño de partícula $0,3-0,5 \mathrm{~mm}$ ). Fuente: Autores

En la Fig. 5c. se aprecia el efecto de la concentración inicial del colorante. Así, a bajas concentraciones $\left(50 \mathrm{mg} \mathrm{L}^{-1}\right)$ el tiempo requerido para alcanzar la saturación es mayor, favoreciendo la retención del AM. Por ejemplo, a un tiempo de 420 min y una concentración de $100 \mathrm{mg} \mathrm{L}{ }^{-1}$, la saturación alcanzada es del $64 \%$, en tanto que solamente alcanza un valor del $4,0 \%$ a 50 $\mathrm{mg} \mathrm{L}{ }^{-1}$. Lo anterior confirma que los cambios en los gradientes de concentración afectan la velocidad de saturación del lecho y el tiempo de ruptura. Este comportamiento se explica debido a la mayor disponibilidad de moléculas de colorante que pueden ser adsorbidas. Por otro lado, este incremento de concentración aumenta la fuerza impulsora en el proceso.

\subsection{Modelo de Adams-Bohart}

Este modelo fue aplicado a los datos experimentales para describir la parte inicial de las curvas de ruptura, Fig. 5. Los parámetros característicos, como la máxima capacidad de adsorción $\left(N_{0}\right)$, la constante cinética $\left(k_{A B}\right)$, el error estadístico $(S S)$ y el coeficiente de correlación $\left(R^{2}\right)$ se muestran en la Tabla 2. Cabe señalar, que el modelo fue aplicado haciendo un ajuste a la curva de ruptura desde su parte su parte inicial, hasta la región de concentración relativa $\mathrm{C}_{\mathrm{t}} / \mathrm{C}_{0}=0,1(10 \%)$.

Los valores de $S S$ (menores de 0,12) y $R^{2}(0,87-0,96)$ señalan un buen ajuste de los datos experimentales a medida que incrementa la altura de columna y disminuye tanto la velocidad de entrada como la concentración inicial de colorante, (ver Tabla 2). Así, el modelo de Adams-Bohart es apropiado para predecir el comportamiento inicial del sistema.

\subsection{Modelo de Thomas}

Se aplicó el modelo de Thomas, mostrado en la Fig. 6, para determinar la constante de velocidad $\left(k_{T H}\right)$ y la máxima capacidad de adsorción $q_{0}\left(\mathrm{mg} \mathrm{g}^{-1}\right)$, a partir de una regresión no lineal, las cuales, junto con el SS y el $\mathrm{R}^{2}$ se presentan en la Tabla 3. 
Y. A. Villada-Villada et al. / Uso de la cascarilla de arroz para la remoción de azul de metileno en columnas de lecho empacado

Tabla 2. Parámetros del Modelo de Adams-Bohart usando regresión no lineal. Fuente: Autores

\begin{tabular}{ccccccc}
\hline $\begin{array}{c}\mathrm{C}_{0} \\
\left(\mathrm{mg} \mathrm{L}^{-1}\right)\end{array}$ & $\begin{array}{c}\mathrm{V} \\
\left(\mathrm{mL} \mathrm{min}^{-1}\right)\end{array}$ & $\begin{array}{c}\mathrm{Z} \\
(\mathrm{cm})\end{array}$ & $\begin{array}{c}\mathrm{k}_{\mathrm{AB}} \\
\left(\mathrm{L} \mathrm{mg}^{-1} \mathrm{~min}^{-1}\right)\end{array}$ & $\begin{array}{c}\mathrm{N}_{0} \\
\left(\mathrm{mg} \mathrm{L}^{-1}\right)\end{array}$ & $\mathrm{R}^{2}$ & $\mathrm{SS}$ \\
\hline 100 & 20 & 10 & 0,000284 & 6562,0 & 0,87 & 0,00 \\
100 & 20 & 15 & 0,000541 & 3846,6 & 0,95 & 0,00 \\
100 & 20 & 20 & 0,000532 & 3846,4 & 0,99 & 0,00 \\
100 & 10 & 20 & 0,000041 & 8501,0 & 0,87 & 0,12 \\
50 & 10 & 20 & 0,000127 & 5000,4 & 0,89 & 0,01 \\
50 & 20 & 20 & 0,000285 & 6430,0 & 0,96 & 0,01 \\
\hline
\end{tabular}

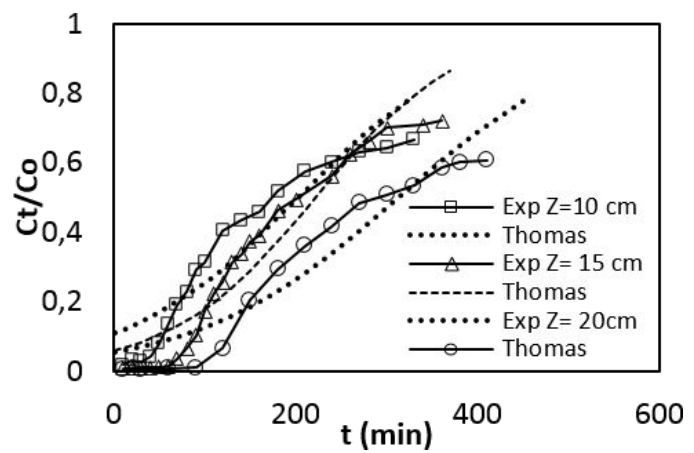

a)

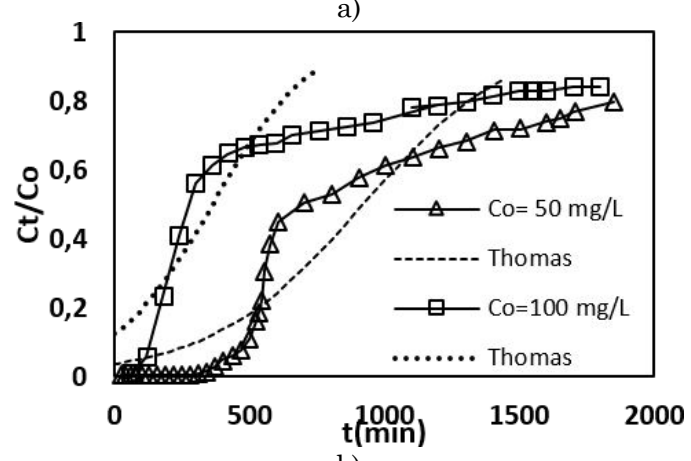

b)

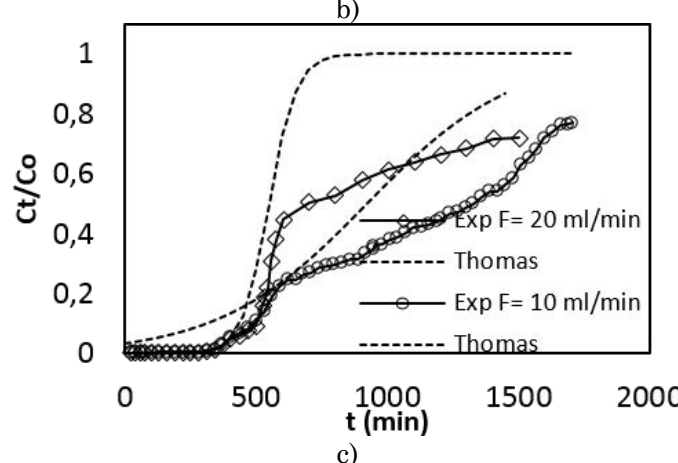

Fig. 6. Curvas de ruptura. Modelo de Thomas aplicado al sistema AM-CA, a) Efecto de la altura, b) efecto de la concentración inicial y c) efecto de la velocidad de entrada ( $\mathrm{pH}=9,0$, tamaño de partícula 0,3-0,5 $\mathrm{mm}$ ).

Fuente: Autores

En ella se aprecia que un incremento en la concentración inicial de colorante conduce a una disminución de los valores de $k_{T H}$ y a un aumento de los valores de $q_{0}$. La fuerza impulsora del proceso de difusión genera un gradiente de concentración entre colorante-adsorbente y colorante-solución, de tal forma que a altas concentraciones, la carga de colorante por gramo de adsorbente, $q_{0}$, es mayor. Con respecto a la velocidad de flujo, se observan resultados opuestos, es decir, flujos mayores incrementan la constante de velocidad, $k_{T H}$ y disminuyen $q_{0}$, sugiriendo que la adsorción de AM sobre CA se ve favorecida a flujos menores.

Los valores de $S S$ se encuentran entre 0,02 y 0,10 , señalando un ajuste aceptable si se considera que 30 puntos experimentales fueron utilizados para el ajuste de cada curva. Por su parte, el $R^{2}$ varía entre 0,82 y 0,94, indicando una buena correlación.

\subsection{Modelo BDST (Bed depth/service time)}

El modelo BDST se basó en la medida de la capacidad del lecho de CA a diferentes valores de ruptura, $(\mathrm{Ct} / \mathrm{Co}=0,2,0,4 \mathrm{y}$ $0,6)$, a diferentes alturas $(10,15$ y $20 \mathrm{~cm})$, a una velocidad de flujo de $20 \mathrm{~mL} \mathrm{~min}^{-1} \mathrm{y}$ concentración inicial de colorante de 100 mg/L, como se muestran en la Fig. 7.

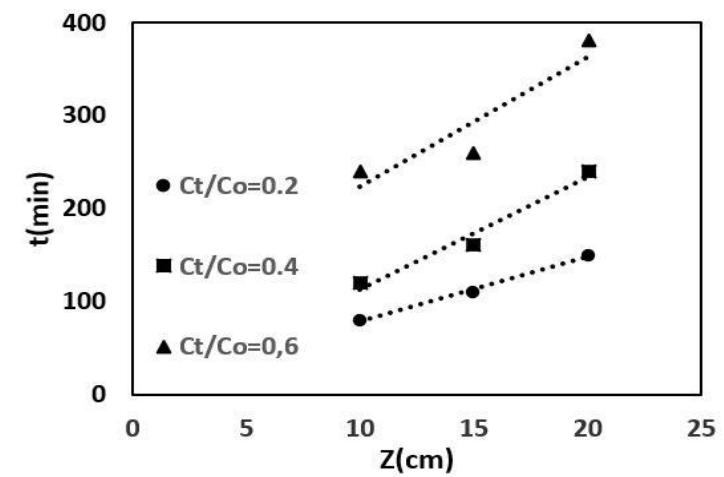

Fig. 7. Rupturas de 0,2, 0,4 y 0,6 para diferentes tamaños de altura a una $\mathrm{Co}=100 \mathrm{mg} / \mathrm{L}$ y una velocidad de entrada de $20 \mathrm{~mL} \mathrm{~min}^{-1}$. Fuente: Autores 
Y. A. Villada-Villada et al. / Uso de la cascarilla de arroz para la remoción de azul de metileno en columnas de lecho empacado

Tabla 3. Parámetros del Modelo de Thomas usando regresión no lineal. Fuente: Autores

\begin{tabular}{ccccccc}
\hline $\begin{array}{c}\mathrm{C}_{0} \\
\left(\mathrm{mg} \mathrm{L}^{-1}\right)\end{array}$ & $\begin{array}{c}\mathrm{V} \\
\left(\mathrm{mL} \mathrm{min}^{-1}\right)\end{array}$ & $\begin{array}{c}\mathrm{Z} \\
(\mathrm{cm})\end{array}$ & $\begin{array}{c}\mathrm{k}_{\mathrm{TH}} \\
\left(\mathrm{mL} \mathrm{min}^{-1} \mathrm{mg}^{-1}\right)\end{array}$ & $\begin{array}{c}\mathrm{q}_{0} \\
\left(\mathrm{mg} \mathrm{g}^{-1}\right)\end{array}$ & $\mathrm{R}^{2}$ & $\mathrm{SS}$ \\
\hline 100 & 20 & 10 & 0,116 & 26,59 & 0,88 & 0,08 \\
100 & 20 & 15 & 0,126 & 21,41 & 0,92 & 0,07 \\
100 & 20 & 20 & 0,089 & 22,24 & 0,92 & 0,06 \\
100 & 10 & 20 & 0,056 & 25,01 & 0,82 & 0,10 \\
50 & 10 & 20 & 0,071 & 17,24 & 0,87 & 0,01 \\
50 & 20 & 20 & 0,431 & 17,04 & 0,96 & 0,02 \\
\hline
\end{tabular}

Las constantes asociadas al modelo de acuerdo con la pendiente e intercepto de cada línea, (a, b, $\left.K_{a}, N_{o}, \mathrm{R}^{2}\right)$ se listan en la Tabla 4.

Tabla 4. Constantes del modelo BDST para la adsorción de $\mathrm{AM}(\mathrm{Co}=100 \mathrm{mg} / \mathrm{L} \mathrm{y} \mathrm{V}=20 \mathrm{~mL} / \mathrm{min})$ Fuente: Autores

\begin{tabular}{cccccc}
\hline $\mathrm{Ct} / \mathrm{Co}$ & $\begin{array}{c}\mathrm{a} \\
\left(\mathrm{min} \mathrm{cm}^{-1}\right)\end{array}$ & $\begin{array}{c}\mathrm{b} \\
(\mathrm{min})\end{array}$ & $\begin{array}{c}\mathrm{K}_{\mathrm{a}} \\
\left(\mathrm{L} \mathrm{mg}^{-1} \mathrm{~min}^{-1}\right)\end{array}$ & $\begin{array}{c}\mathrm{N}_{0} \\
\left(\mathrm{mg} \mathrm{L}^{-1}\right)\end{array}$ & $\mathrm{R}^{2}$ \\
\hline 0,2 & 7 & 8,33 & 0,001585311 & 2979,91 & 0,99 \\
0,4 & 12 & 6,66 & 0,000607814 & 5108,42 & 0,96 \\
0,6 & 14 & 83,33 & 0,000053431 & 5898,22 & 0,96 \\
\hline
\end{tabular}

Se observa que cuando los valores de $\mathrm{Ct} / \mathrm{Co}$ se incrementan, la constante de velocidad $K_{a}$ decrece y la capacidad del lecho por unidad de volumen $\mathrm{N}_{0}$ aumenta. Esto se debe a que, $K_{a}$ está asociada directamente con la velocidad de transferencia de masa del colorante al adsorbente, así una menor $K_{a}$ genera una reducción en la velocidad de transferencia y con ello, un mayor tiempo de contacto entre la solución de AM y CA. Lo anterior, permite una mayor remoción de las moléculas de colorante sobre la superficie del adsorbente.

Finalmente, los valores de $\mathrm{R}^{2}(0,96$ $0,99)$ muestran el ajuste y la validez del método, indicando que la variable altura del lecho es fundamental en la adsorción en continuo y pueden usarse como preámbulo en el proceso de escalado.

\section{CONCLUSIONES}

Los resultados confirman que la CA constituye un adsorbente adecuado para la remoción eficiente del colorante catiónico
AM mediante lechos empacados. El proceso de adsorción en continuo se ve favorecido a $\mathrm{pH}$ básico, $\mathrm{pH}=9,0$, tamaño de partícula entre $0,3 \mathrm{~mm}<x<0,5 \mathrm{~mm}$, concentración inicial de colorante de $50 \mathrm{mg} \mathrm{L}^{-1}$, flujo de 10 $\mathrm{mL} \mathrm{min}^{-1}$ y altura de columna de $20 \mathrm{~cm}$. Estas condiciones incrementan el tiempo requerido para alcanzar la saturación, permitiendo que una mayor cantidad del colorante sea retenido en la columna.

El diseño de cribado $2^{3}$ permitió establecer la correlación de los factores evaluados en la remoción en continuo del sistema AM-CA, estableciendo que la concentración inicial de colorante y la altura del lecho fueron los factores de mayor significancia. Por otro lado, la velocidad de entrada y su interacción con la altura y la concentración inicial de colorante mostraron ser factores no representativos en la variable de respuesta, correspondiente al porcentaje de remoción del colorante.

Los resultados de los modelos matemáticos implementados muestran que el modelo de Adams-Bohart ofrece un buen ajuste para el comportamiento dinámico de la parte inicial de la curva de ruptura. Así mismo, los modelos de Thomas y BDST señalan una correlación adecuada con los datos experimentales para el proceso de remoción del AM.

\section{AGRADECIMIENTOS}

Los autores expresan agradecimientos a la Universidad Nacional de Colombia Sede Medellín por la dotación en la infraestructura del Laboratorio de Química 
Experimental. Por la financiación de esta investigación a Colciencias mediante el otorgamiento de la Beca - Pasantía de Joven Investigador e Innovador año 2012, Virginia Gutiérrez de Pineda. También agradecen a CONICET, Argentina, por su contribución financiera.

\section{REFERENCIAS}

[1] G. E. Walsh, L. H. Bahner, and W. B. Horning, "Toxicity of textile mill effluents to freshwater and estuarine algae, crustaceans and fishes," Environ. Pollut. Ser. A, Ecol. Biol., vol. 21, no. 3, pp. 169-179, Mar. 1980.

[2] N. Kannan and M. M. Sundaram, "Kinetics and mechanism of removal of methylene blue by adsorption on various carbons-a comparative study," Dye. Pigment., vol. 51, no. 1, pp. 25-40, Oct. 2001.

[3] G. Crini, "Non-conventional low-cost adsorbents for dye removal: a review.," Bioresour. Technol., vol. 97, no. 9, pp. 1061-85, Jun. 2006.

[4] M. Rafatullah, O. Sulaiman, R. Hashim, and A. Ahmad, "Adsorption of methylene blue on low-cost adsorbents: a review.," J. Hazard. Mater., vol. 177, no. 1-3, pp. 70-80, May 2010.

[5] V. Vadivelan and K. V. Kumar, "Equilibrium, kinetics, mechanism, and process design for the sorption of methylene blue onto rice husk.," J. Colloid Interface Sci., vol. 286, no. 1, pp. 90-100, Jun. 2005.

[6] A. Moreno, D. Figueroa, and A. Hormaza, "Diseño estadístico para la remoción eficiente del colorante rojo 40 sobre tusa de maíz," Prod. + Limpia, vol. 7, no. 2, pp. 9-19, 2012.

[7] A. Hormaza, D. Figueroa, and A. Moreno, "Evaluación de la remoción de un colorante azo sobre tuza de maíz mediante diseño estadístico," Rev. la Fac. Ciencias, vol. 1, no. 1, pp. 61-71, 2012.

[8] C. Namasivayam, D. Prabha, and M. Kumutha, "Removal of direct red and acid brilliant blue by adsorption on to banana pith," Bioresour. Technol., vol. 64, no. 1, pp. 77-79, Apr. 1998.

[9] A. C. Jaramillo, A. M. Echavarría, and A. Hormaza, "Diseño Box-Behnken para la optimización de la adsorción del colorante azul ácido sobre residuos de flores," Ing. y Cienc., vol. 9, no. 18, pp. 75-91, 2013.

[10] W. S. Alencar, E. Acayanka, E. C. Lima, B. Royer, F. E. de Souza, J. Lameira, and C. N. Alves, "Application of Mangifera indica (mango) seeds as a biosorbent for removal of Victazol Orange 3R dye from aqueous solution and study of the biosorption mechanism," Chem. Eng. J., vol. 209, pp. 577-588, Oct. 2012.

[11] M. Doria H., A. Hormaza A., and D. Gallego S., "Cascarilla de arroz: material alternativo y de bajo costo para el tratamiento de aguas contaminadas con cromo (VI)," Rev. Gestión y Ambiente, vol. 14, no. 1, pp. 73-84, 2011.

[12] T. G. Chuah, A. Jumasiah, I. Azni, S. Katayon, and S. Y. Thomas Choong, "Rice husk as a potentially lowcost biosorbent for heavy metal and dye removal: an overview," Desalination, vol. 175, no. 3, pp. 305-316, May 2005.

[13] P. K. Malik, "Use of activated carbons prepared from sawdust and rice-husk for adsorption of acid dyes: a case study of Acid Yellow 36," Dye. Pigment., vol. 56, no. 3, pp. 239-249, Mar. 2003.

[14] J. Sharma and B. Janveja, "A study on removal of Congo red dye from the effluents of textile industry using rice husk carbon activated by steam," Rasayan J. Chem., vol. 1, no. 4, pp. 936-942, 2008.

[15] R. Han, Y. Wang, W. Yu, W. Zou, J. Shi, and H. Liu, "Biosorption of methylene blue from aqueous solution by rice husk in a fixed-bed column.," J. Hazard. Mater., vol. 141, no. 3, pp. 713-8, Mar. 2007.

[16] R. Han, D. Ding, Y. Xu, W. Zou, Y. Wang, Y. Li, and L. Zou, "Use of rice husk for the adsorption of congo red from aqueous solution in column mode.," Bioresour. Technol., vol. 99, no. 8, pp. 2938-46, May 2008.

[17] R. Han, Y. Wang, X. Zhao, F. Xie, J. Cheng, and M. Tang, "Adsorption of methylene blue by phoenix tree leaf powder in a fixed-bed column: experiments and prediction of breakthrough curves," Desalination, vol. 245, no. 1-3, pp. 284-297, Sep. 2009. 